\title{
Human Caveolin-1 a Potent Inhibitor for Prostate Cancer Therapy: A Computational Approach
}

\section{List of authors in order of their authorship}

Uzma Khanam $^{1}$, Pallavi Somvanshi ${ }^{2 \#}$, Shafiul Haque ${ }^{3,4}$, Balwant Kishan Malik ${ }^{5}$, Bhawna Rathi $^{1 *}$

\section{Authors' affiliations}

${ }^{1}$ Centre for Computational Biology and Bioinformatics, Amity Institute of Biotechnology, Amity University (Noida Campus), Noida-201301, India

2 Department of Biotechnology, TERI University, 10 Institutional Area, Vasant kunj, New Delhi-110070, India

${ }^{3}$ Department of Biosciences, Jamia Millia Islamia (A Central University), New Delhi-110025, India

${ }^{4}$ Research \& Scientific Studies Unit, College of Nursing \& Allied Health Sciences, Jazan University, Jazan-45142, Saudi Arabia

${ }^{5}$ Department of Biotechnology, Sharda University, Greater Noida-201306, India

\section{Corresponding Author(s):}

*First Corresponding Author: Dr. Bhawna Rathi,

Centre for Computational Biology and Bioinformatics,

Amity Institute of Biotechnology,

Amity University (Noida Campus),

Noida-201301, India

Phone: +91-9953840456

Email:brathi@amity.edu

\#Second Corresponding Author: Dr. Pallavi Somvanshi,

Department of Biotechnology,

TERI University,

10, Institutional Area, Vasant Kunj

New Delhi-110070, India

Phone: +91-9899931682

Email: psomvanshi@gmail.com 


\begin{abstract}
Caveolin-1 (Cav-1) is $22 \mathrm{kDa}$ caveolae protein, acts as a scaffold within caveolar membranes. It interacts with alpha subunits of G-protein and thereby regulates their activity. Earlier studies reported elevated or up-regulated levels of caveolin-1 in the serum of prostate cancer patients. Secreted Cav-1 promotes angiogenesis, cell proliferation and anti-apoptotic activities in prostate cancer patients. Cav-1 upregulation is mainly related to prostate cancer metastasis. Keeping above facts in view, the present study was designed to explore Cav-1 as a target for prostate cancer therapy using computational approach. Molecular docking, structural base molecular modelling and molecular dynamics simulations were performed to investigate Cav1 inhibitors. A predictive model was generated and validated to establish a stable structure. ZINC database of biogenic compounds was used for induced fit docking (IFD) and high throughput virtual screening. The H-bond interactions of the compounds with active site residues of Cav-1 were estimated by IFD and $100 \mathrm{~ns}$ long molecular dynamic simulations. The reported compounds showed significant binding and thus can be considered as potent therapeutic inhibitors of Cav-1. This study provides a valuable insight into biochemical interactions of Cav-1 for therapeutic applications and warrants for experimental validation of the predicted 'active(s)'.
\end{abstract}




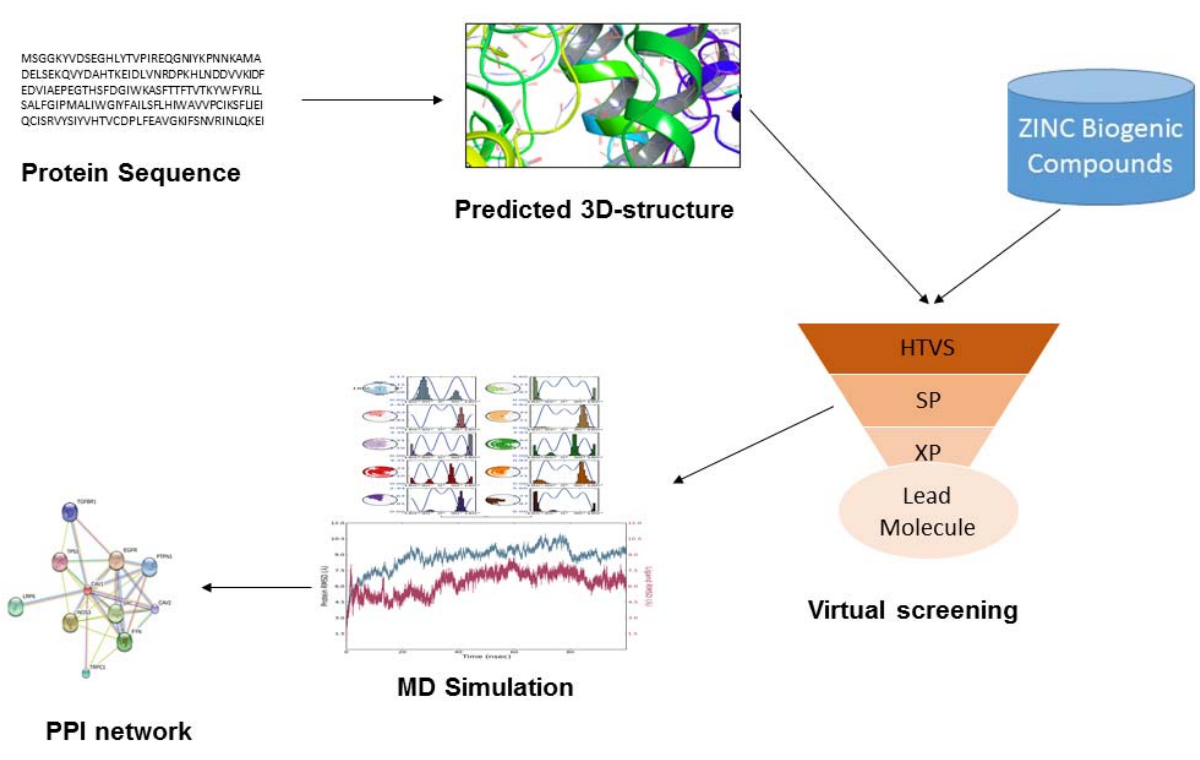

Keywords: molecular dynamics simulation; virtual screening; molecular docking; prostate cancer; caveolin-1

\section{Abbreviations}

Cav-1 Caveolin-1

IFD Induced fit docking

MD Molecular dynamics

VSW Virtual screening workflow

STRING Search Tool for the Retrieval of Interacting Genes

SP Standard precision

XP Extra precision 
HTVS High throughput virtual screening

RMSD Root mean-square deviation

RMSF Root mean-square fluctuation

PPI Protein-protein interaction

TGFBR1 Transforming growth factor beta receptor 1

PTPN1 Protein tyrosine phosphatase, non-receptor type 1

TRPC1 Transient receptor potential cation channel 1

LRP6 Low density lipoprotein receptor-related protein 6

SRC V-src sarcoma (Schmidt-Ruppin A-2) viral oncogene homolog

EGF Epidermal growth factor

$\mathrm{NOS}_{3} \quad$ Nitric oxide synthase 3

\section{Introduction}

Prostate cancer is the third most common cancer and the second leading cause of death in males in the western countries ${ }^{1}$, and surgery or radiotherapy is generally used to treat prostate adenocarcinoma. This non-localized disease generally erupts during the course of androgen suppression at the start of the primary therapy ${ }^{2}$. Till now, not a single effective therapeutic strategy is available after the failure of the primary treatment. Earlier studies have identified a large number of candidate genes involved in prostate cancer metastasis ${ }^{3}$. Caveolin-1 (Cav-1) has been found upregulated in mouse cell lines with metastatic prostate cancer ${ }^{4}$. Cav-1 is a 21 - 
$24 \mathrm{kDa}$ multi-functional signalling protein and lipid transporter ${ }^{5}$. Caveolae, which are specialized plasma membrane invaginations, found in smooth muscle cells, adipocytes and endothelium, comprises Cav-1 as its major structural component ${ }^{6}$. Cav-1 mediated oncogenic mechanisms in prostate cancer involves two pathways, first, interaction of enhanced Cav-1 expression with cellular signalling factors to stimulate the proliferation and activation of antiapoptotic mechanisms ${ }^{7}$. Also, PI3K/Akt pathway remains constantly active in cultured prostate cancer cells because of the binding of upregulated Cav-1 to protein phosphatases PP1 and $\mathrm{PP} 2 \mathrm{~A}^{8}$. The progression and development of prostate tumors is significantly reduced in Cav-1 negative mice, and prostatic hyperplasia is caused in prostatic epithelial cells due to overexpression of Cav-1 ${ }^{9}$. Second, Cav-1 secreted by the prostate cancer cells into the tumor micro-environment promotes the cell growth and angiogenesis ${ }^{10,11}$. Elevated Cav-1 in the metastasis-derived cells suggests that Cav-1 is correlated with prostate cancer metastasis ${ }^{8,9}$.

Cav-1 has been found as a potential therapeutic target for the prostate cancer metastasis ${ }^{12}$. Unfortunately, almost all the therapies of prostate cancer are associated with various side effects. Natural compounds have been found very effective from ancient time to treat various types of cancers and warrants for the identification of therapeutic natural compounds against prostate cancer.

As the drug discovery process is exhaustive, labour intensive, expensive and time consuming, in silico studies offer a cost effective platform for the discovery of 'active' molecules. In- silico analysis was performed earlier using different therapeutic targets ${ }^{13}$.

In the present study, in silico analyses were performed to discover natural compounds as Cav1 inhibitor. Although, the crystal structure of Cav-1 has not been yet resolved. However, protein structure predictions were done to perform the current study. Virtual screening and induced fit docking (IFD) were performed for the identification of Cav-1 inhibitors. Molecular dynamics (MD) simulation was carried out to decipher the interaction pattern and stability of 
ligand-Cav-1 complex. Protein-protein interaction (PPI) network of Cav-1 was generated for the elucidation of the interacting proteins. Overall, the present study offers a sturdy platform for the prospective experimental validations of Cav-1 inhibitor(s) for therapeutic applications against prostate cancer based on the predictive findings obtained from this potential in silico study.

\section{Results and Discussion}

\section{Protein Structure Prediction and Validation}

BLAST search was performed for human Cav-1 (178 amino acid) in order to search the suitable template for the structure prediction as done in other similar studies executed in bacterial disease $^{14}$. Based on the results of BLAST analysis, sequence similarity was observed too low for the homology modelling, so structure prediction was done using I-Tasser server. Structure validation of the generated model showed a good quality of the predicted model (Table 1). 3Dstructure of the predicted model is shown in Figure 1. Ramachandran plot analysis suggested that the predicted model has $93.1 \%$ residues in the most favoured regions indicating the good quality of the model, as a good quality model is supposed to have more than $90 \%$ of its residues in the most favoured regions. The generated model passed through PROVE program with 1.631 Z-score RMS. Overall ERRAT (protein structure verification algorithm well suited for evaluation of crystallographic model building and refinement) quality factor value was $72.95 \%$. All validation results strongly favoured the acceptance reliability of the predicted 3D model.

\section{Active Site Prediction}

Active site(s) were predicted using Sitemap. Two drug able sites were generated with good site scores (0.912 and 0.898). Actives site having site score 0.912 were selected for further docking studies.

\section{Molecular Docking and Virtual Screening}


A recent study demonstrated that virtual screening is more significant than high-throughput screening because of less time consumption and cost effectiveness ${ }^{15,16}$. Receptor-based virtual screening for various targets including proteases has demonstrated the success rate of in silico studies $^{17}$. The structure-based virtual screening methodology remains highly desirable for the identification of novel compounds with structural diversity, as the active site of the protein serves as a $\operatorname{target}^{18}$. In the present study, ZINC compounds optimized with the OPLS-2005 force field were subjected to ADME filters to reduce the false-positives and to avoid poor pharmacokinetic profiles. In addition, Lipinski rule of five was calculated applying cut-off range of the removal of the outliers ${ }^{19}$. By strictly following the above mentioned criteria and filters, the Zbc library was compressed to 164530 compounds, which were then subjected to a stepwise high throughput virtual screening (HTVS) protocol ${ }^{20}$. Initially, the standard precision (SP) and extra-precision (XP) docking were performed to calculate the Glide score and Glide energy. Figure 2 illustrates the $2 \mathrm{D}$ conformation of the ligands with the top-five highest scores obtained after XP docking. The top-five compounds were used for IFD in order to calculate the best pose. Hydrogen-bond interactions were noted between the top-five hit compounds and the active site residues (Figure 3). Out of all the hit compounds, ZINC13410492 showed the highest Glide score $(-8.032 \mathrm{kcal} / \mathrm{mol})$, Glide energy $(-40.244 \mathrm{kcal} / \mathrm{mol})$ and IFD score ($339.94 \mathrm{kcal} / \mathrm{mol}$ ) (Table 2), with two hydrogen bonds and one salt bridge formed with the binding pocket residues of Cav-1. The nitrogen atom of the ligand attached to form a hydrogen bond with the oxygen atom of Asp 169 (Lig: NH2 $\cdots$ Asn, 169, $2.38 \AA$ ) and another hydrogen bond was formed between the nitrogen atom of ligand and oxygen atom of Glu 69

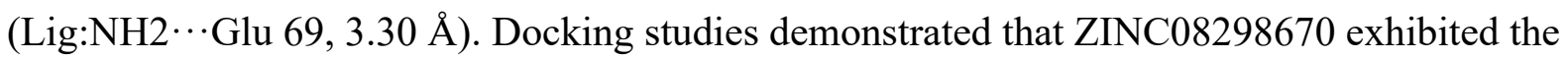
second highest Glide score (-7.62 kcal/mol), Glide energy $(-42.47 \mathrm{kcal} / \mathrm{mol})$, and IFD score $(-337.91 \mathrm{kcal} / \mathrm{mol})$ (Table 2), with the formation of a single hydrogen bond between the nitrogen atom of the ligand and the oxygen atom of Glu 69 present in the binding cavity of 
Cav-1. It also showed single pi-pi stacking between the ligand and Phe 99, and a salt bridge with Glu 69. Compound ZINC13550046 demonstrated the formation of four hydrogen bonds and a single pi-cation interaction between the ligand and Cav-1, and exhibited the third highest Glide score $(-7.152 \mathrm{kcal} / \mathrm{mol})$, Glide energy $(-36.098 \mathrm{kcal} / \mathrm{mol})$, and IFD score $(-333.94$ $\mathrm{kcal} / \mathrm{mol})$. Compound ZINC01535903 demonstrated the formation of three hydrogen bonds and a single pi-cation interaction between ligand and Cav-1, and exhibited the fourth highest Glide score $(-7.052 \mathrm{kcal} / \mathrm{mol})$, Glide energy $(-45.35 \mathrm{kcal} / \mathrm{mol})$ and IFD score $(-342.45$ $\mathrm{kcal} / \mathrm{mol}$ ). Compound ZINC13431926 demonstrated the formation of two hydrogen bonds between the ligand and Cav-1, and exhibited the fifth highest Glide score $(-6.978 \mathrm{kcal} / \mathrm{mol})$, Glide energy $(-35.302 \mathrm{kcal} / \mathrm{mol})$, and IFD score $(-331.89 \mathrm{kcal} / \mathrm{mol})$. Glide score has been proved as a reliable scoring function for the screening of databases, with steady performance against pharmaceutically relevant protein-ligand complexes, like HIV-1 protease and p38 MAP kinase (16). Hence, in the present study, we have ranked the inhibitors based on their glide score.

\section{Binding Energy Calculation}

In MM/GBSA score analysis was performed to determine the binding efficiency using the pose viewer file of the protein-ligand complex generated from XP docking. The estimated efficiencies of the hit compounds ZINC13410492, ZINC08298670, ZINC13550046, ZINC01535903 and ZINC13431926 were found to be $-63.345,-58.716,-46.152,-55.253$ and$59.28 \mathrm{kcal} / \mathrm{mol}$, respectively (Table 2 ).

\section{Protein-Ligand Complex Simulation}

During the flexible docking, protein receptor was considered rigid in nature. To investigate the dynamic stability and interaction pattern of the ligand-protein complex, molecular dynamics simulation was performed for 100 ns. ZINC13410492-Cav-1 complex having highest Glide score was considered for this simulation study. Conformational links of the system were 
monitored using the root mean-square deviation (RMSD) values of C-alpha atoms. Figure 4 shows the RMSD values of ZINC13410492 system for 100 ns MD simulation. Trajectories analysis revealed that ZINC13410492 system was stable and well within the binding pocket of Cav-1. Lower root mean-square fluctuation (RMSF) values were depicted by the residues of the active site and alpha helix regions, suggested the stability of the regions (Figure 5). Analysis of the post-processing interaction was done to examine the ligand-protein binding interactions by implementing the trajectory frames generated from the MD simulation. The system stability was maintained mainly due to the hydrogen bond interaction with the active site residue Glu 69 in the $67 \%$ of the simulation time, for rest of the time Glu 69 was engaged in the formation of salt and water bridges (Figure 6). The constructed hydrogen bond with Asn 169 from the docking studies was not stable during the MD simulation. Other residues demonstrated hydrophobic contacts with Cav-1 viz. Phe 68, Leu 102, Ala 105, Leu 138, Ilu 139, Cys 135, Arg 146, Val 147, Val 170, and Ilu 172.

\section{ADMET (absorption, distribution, metabolism and excretion-toxicology) analysis}

ADMET analysis of the selected compounds was done by QikProp module of the Schrodinger package (Table 3). Out of the top-five selected compounds, ADMET parameters of ZINC13410492 (highest score) has been illustrated here. \#star represents the properties lying outside the similarity criteria of $95 \%$ of the known drugs with reference range between 0 to $5^{21}$. The star value 1 for ZINC13410492 suggested that only 1\% molecular properties of the compound drops out of the reference range. CNS property between -2 to 2 (activity of central nervous system) was found to be 0 for this compound. QPlogBB value for predicted blood brain partition coefficient was found to be -0.404 , which falls under the reference range of 3.0 to 1.2. The reference range of SASA (solvent accessible surface area), FISA (hydrophilic component of SASA) and (hydrophobic component of SASA) were 300 to 1000, 7 to 330, and 0 to 750 , respectively, and predicted that the values of the given parameters falls between the 
standard reference range. The predicted value of percent human oral absorption was $89.386 \%$. The predicted value of skin permeability factor (QPlogkp) was found to be -4.733 , which was also within the given reference range -8 to 1.0 . The compound (ZINC13410492) showed high value of the coefficient for the parameters, QPlogPC16 (solvation free energy in hexadecane) having value 14.91 (reference range 4 to 18 ), QPlogPw (solvation free energy in water) with the value of 9.698 (reference range 4 to 45), and QPlogPoct (solvation free energy in octanol) having value 20.598 (reference range 8 to 35). Easy absorption of the compound by the lipid bilayer was predicted by $\mathrm{QP} \log \mathrm{Po} / \mathrm{w}$ (octanol/water partition coefficient) value of -4.793 (reference range -2 to 6.5$)$. The values for ionization potential and electron linkage (eV) were found to be 9.283 (reference range 7.9 to 10.5 ) and -0.217 (reference range -0.9 to 1.7 ), respectively. Also, the compound (ZINC13410492) was found to be satisfactory according to Jorgensen rule of three infringement and Lipinski rule of five.

\section{Protein-Protein Interaction Network}

The PPI network was constructed with 11 nodes and 32 edges based on the information available in the STRING database, where the nodes represents the proteins and the connection between the nodes reflects the number of possible interactions between the proteins ${ }^{22}$. Hub nodes were considered as high degree nodes due to their high connectivity. Interacting proteins in the PPI network were, viz. transforming growth factor beta receptor 1 (TGFBR1), protein tyrosine phosphatase non-receptor type 1 (PTPN1), Transient receptor potential cation channel 1 (TRPC1), Low density lipoprotein receptor-related protein 6 (LRP6), FYN oncogene, v-src sarcoma (Schmidt-Ruppin A-2) viral oncogene homolog (SRC), Epidermal Growth Factor (EGFR), nitric oxide synthase $3\left(\mathrm{NOS}_{3}\right)$, and tumor protein p53. Figure 7 showed that the hub nodes in the PPI network were EGFR (degree $=8)$, FYN (degree $=8)$, SRC (degree = 7), FRY $($ degree $=8)$ and NOS3 (degree $=6)$. All the above mentioned proteins directly interact with Cav-1 and control the different signalling pathways. TGFBR1 plays a significant role in the 
cellular differentiation and the attenuation of TGFBR1 by Cav-1 is an important mechanism to control the progression of the developmental events. PTPN1 is localized in caveolae membrane and directly interacts with Cav-1, and controls the EGF and SRC family. Cav-1 interacts with the wild type SRC family kinases and suppresses the auto-activation of FYN. Cav-1 acts as a membrane adaptor to link the $\mathrm{ERK}^{23}$ and the tyrosine kinase FYN, and interferes with the cell growth. Cav-1 and $\mathrm{NOS}_{3}$ interaction plays a major role in normal endothelial $\mathrm{NOS}_{3}$ activity and vascular bioavailability of nitric oxide.

In conclusion, Cav-1 is a potential target for anti-cancer drug designing and virtual screening can act as a potential step towards the development of new therapy against prostate cancer. Novel inhibitors were identified by in silico screening of biogenic compounds from the ZINC database using induced fit docking programs. Strong binding affinity of the selected compounds was predicted for Cav-1 by docking studies. The selected compound (ZINC13410492) was further validated by MD simulations. Cav-1 has been found an important protein which involve in a number of signalling pathways to control the cell progression. Overall, our current in silico findings offers a solid background for the future experimental validations of Cav-1 inhibitor(s) for prostate cancer therapy.

\section{Material and Methods}

\section{Protein Structure Prediction and Validation}

Due to non-availability of the three dimensional (3D) structure of Cav-1 in the protein data bank $(\mathrm{PDB})^{24}$, the 3D-structure of Cav-1 was generated from the primary sequence retrieved from UniProt knowledge database. The primary sequence of human Cav-1 was extracted (UniProt Id: Q03135) and the sequence was searched against PDB entries using protein-protein BLAST to identify the template crystal structure. The 3D-structure of Cav-1 was predicted by I-Tasser server (Iterative Threading Assembly Refinement), based on multiple threading 
approach followed by full-length atomic model construction employing iterative template fragment assembly simulations ${ }^{25-28}$. The quality of the generated model was evaluated by using PROCHECK ${ }^{29}$, PROVE $^{30}$ and ERRAT ${ }^{31}$ programs.

\section{Protein Preparation and Structure Refinement}

The modelled 3D structure of Cav-1 was prepared by using protein preparation wizard of the Schrodinger Suite ${ }^{32}$ to ensure the quality and reliability of the structure. Protein optimization and minimization was done by employing OPLS (Optimized Potentials for Liquid Simulations) 2005 force field.

\section{Active-Site Prediction}

Sitemap was employed to predict the interacting residues as it provides indications and pertinent information about the suitable position for the donor or the acceptor or the hydrophobic group in the receptor. The physical parameters selected for describing the drug ability included hydrophilicity, degree of exposure, hydrophobicity, hydrogen bonding and binding site points.

\section{High Throughput Virtual Screening}

ZINC biogenic compounds (Zbc), a commercially available primary and secondary metabolite database, originally includes 189466 compounds was prepared by using OPLS 2005 force field $^{33}$. Zbc compounds have been used to identify the mitogen activated protein kinase inhibitors $^{34}$. The $3 \mathrm{D}$-structures of the small molecules were prepared by using LigPrep 3.5 module before the docking to obtain different stereochemical, tautomeric, and ionization conformer with minimum energy state of the ligands $s^{35,36}$. The prepared database was subjected for the virtual screening workflow (VSW). Prior to the VSW, receptor grid was generated using Glide 6.8 module $^{37}$. The prepared 3D structure of the target protein was used to generate the glide scoring grid for the successive docking calculations. In the VSW, ADME (absorption, distribution, metabolism and excretion) properties were calculated using Qikprop 4.5 module. 
In general, the ADME descriptors ${ }^{38}$ are calculated at the last stage of the drug discovery process, but in the present study, the ADME properties calculations were performed in the preliminary stage to save the time as well as to curtail the cost involved in the process.

\section{Binding Free Energy Calculation}

Generally, the calculations of binding energy are termed to be accurate when compared to the docking energy calculations ${ }^{39}$. Therefore, in the present study, the MMGBSA algorithm in the Prime 4.1 module was employed to determine the binding energy ${ }^{40}$. The equation for the binding energy calculation is:

$$
\mathrm{G}_{\text {bind }}=\Delta \mathrm{E}+\Delta \mathrm{G}_{\mathrm{solv}}+\Delta \mathrm{G}_{\mathrm{SA}}
$$

where ${ }_{2} \Delta \mathrm{E}=\mathrm{E}_{\text {complex }}-\mathrm{E}_{\text {protein }}-\mathrm{E}_{\text {ligand }}$

Above, $E_{\text {complex }}, E_{\text {protein }}$ and $E_{\text {ligand }}$ and shows the minimized energy values of the protein-ligand complex, protein only, and ligand only, respectively. The electrostatic solvation energy of the complex is represented as $\Delta$ Gsolv. Similarly, the nonpolar contribution by the surface area to the solvation energy is represented as $\triangle \mathrm{GSA}$.

\section{Induced-Fit Docking}

In order to avoid the misleading results that might be obtained from the standard docking protocol, IFD was performed by using Glide and Prime modules of Schrodinger Suite for the top five hit compounds obtained from XP docking. The vdW radii having value of 0.5 was used for non-polar atoms of the ligand and receptor, having partial atomic charge less than 0.15 and 0.25 for the ligand and receptor, respectively. Prime refinement was done for the residues present within $5 \AA$ range from the ligand. The best complex was re-docked with Glide XP.

\section{Molecular Dynamics Simulation}

Prior to MD simulation, XP docked complexes were prepared in the same manner as prepared earlier for the virtual screening. MD simulations were performed to obtain the most stable conformation of Cav-1 and ligand complex. Desmond 3.1 MD package ${ }^{41}$ was employed for 
MD simulations using OPLS-AA (All-Atom) force field parameters. An orthorhombic box with periodic boundary conditions was used to solvate the protein by adding SPC (simple point charge model) water molecules. DPPC (dipalmitoyl phosphatidylcholine) lipid bilayer was added and the protein-ligand complex was energy minimized with OPLS-AA force field parameters. The energy minimized complex was subjected to steepest descent method for obtaining maximum force smaller than $1000 \mathrm{~kJ} / \mathrm{mol} \cdot \mathrm{nm}$. Prior to thermalization, the entire system directed for energy minimization upto 1000 steps. Isothermal isobaric (NPT) ensemble was run after the thermalization at a constant temperature of $300 \mathrm{~K}$ and pressure of 1.01325 bar. Nose-Hover chain thermostat ${ }^{42}$ and Martyna-Tobias-Klein barostat ${ }^{43}$ were used to maintain the system. $\mathrm{SHAKE}^{44}$ algorithm was applied for 2-fs time step. Long-range electrostatics interactions were treated by Particle mesh ewald method. Energies and their coordinates for the OPLS-2005 force field simulations were recorded for 2.4 ps for the total $100 \mathrm{~ns}$ for the systems.

\section{Protein-Protein Interaction Analysis}

STRING (Search Tool for the Retrieval of Interacting Genes) was used to create the PPI network of Cav-1.

\section{Acknowledgements}

The authors are thankful to Dr. Puniti Mathur, Coordinator, Centre for Computational Biology and Bioinformatics, and Amity Institute of Biotechnology, Amity University Noida Campus, UP India, for providing the infrastructural support and dry-lab facility for this research study.

\section{Financial statement}

No specific financial support was available for this research work.

\section{Authors' contributions}


Conceived and designed the study and experiments: UK, BKM, SH, PS, BR. Performed the experiments: UK, BKM, BR. Analysed the data: UK, SH, PS, BR. Contributed reagents/materials/analysis tools: $\mathrm{SH}, \mathrm{PS}, \mathrm{BR}$. Wrote the paper: UK, BKM, SH, PS, BR. All authors reviewed the manuscript.

\section{Additional Information}

\section{Competing Financial Interests Statement}

The authors declare no competing financial interests exist.

\section{References}

1. Siegel, R., Miller, K., \& Jemal, A. Cancer Statistics. CA Cancer J Clin, 2016; 66, 7-30.

2. Linton, K. D., \& Hamdy, F. C. Early diagnosis and surgical management of prostate cancer. Cancer Treat. Rev. 2003; 29, 151-160.

3. Harder, T., \& Simons, K. Caveolae, DIGs, and the dynamics of sphingolipidcholesterol microdomains. CurrOpin Cell Biol, 1997; 9, 534-542.

4. Yang, G., Truong, L. D., Timme, T. L., Ren, C., Wheeler, T.M., Park, S. H., Nasu, Y., Bangma, C. H., Kattan, M. W., Scardino, P. T., \& Thompson, T. C. Elevated expression of caveolin is associated with prostate and breast cancer. Clin Cancer Res, 1998; 8, 1873-80.

5. Parton, R. G., Hanzal-Bayer, M., \& Hancock, J. F. Biogenesis of caveolae: A structural model for caveolin-induced domain formation. Journal of Cell Science, 2006; 119, 787 796. doi:10.1242/jcs.02853.

6. Couet, J., Li, S., Okamoto, T., Ikezu, T., \& Lisanti, M. P. Identification of peptide and protein ligands for the caveolin-scaffolding domain: Implications for the interaction of caveolin with caveolae-associated proteins. J Biol Chem, 1997; 272, 6525-6533.

7. Liu, P., Rudick, M., \& Anderson, R. G. W. Multiple Functions of Caveolin-1. 2002; DOI 10.1074/jbc.R200020200

8. Hall, S. J., \& Thompson, T. C. Spontaneous metastatic activities but not experimental metastatic activities differentiate primary tumor derived versus metastasis-derived mouse prostate cancer cell lines. Clin. Exp. Metastasis, 1997; 15, 484-493.

9. Tahir, S. A., Yang, G., Ebara, S., Timme, T. L., Satoh, T., Li, L., Goltsov, A., Ittmann, Michael., Morrisett, J. D., \& Thompson, T. C. Secreted caveolin-1 stimulates cell survival/clonal growth and contributes to metastasis in androgen-insensitive prostate cancer. Cancer Research, 2001; 61, 3882-3885.

10. Ayala, G., Morello, M., Frolov, A., You, S., Li, R., Bartolucci, G., Danza, G., Adam, R.M., \& Timothy, C. Loss of caveolin-1 in prostate cancer stroma correlates with reduced relapse-free survival and is functionally relevant to tumour progression 2013; 231, 77-87. doi:10.1002/path.4217.

11. Williams, T.M., \& Lisanti, M.P. Caveolin-1 in oncogenic transformation, cancer, and metastasis. Am. J. Physiol. Cell Physiol. 2005; 288, C494-C506. doi:10.1152/ajpcell.00458.2004

12. Thompson, T.C., Tahir S.A., Li, L., Watanabe, M., Naruishi, K., Yang, G., Kadmon, D., Logothetis, C.J., Troncoso, P., Ren, C., Goltsov, A., \& Park, S. The role of caveolin1 in prostate cancer: clinical implications. Prostate Cancer Prostatic Disease, 2010; 13, 6-11. doi:10.1038/pcan.2009.29 
13. Khan S Ahmad K, Alshammari E M A., Adnan M, Baig MH, Lohani M, Somvanshi P, Haque $\mathrm{S}$ Implication of Caspase-3 as a Common Therapeutic Target for Multineurodegenerative Disorders and Its Inhibition Using Nonpeptidyl Natural Compounds. BioMed Research International; 2015:379817.

14. Singh V and Somvanshi P Homology modelling of 3-oxoacyl-acyl carrier protein synthase II from Mycobacterium tuberculosis $\mathrm{H} 37 \mathrm{Rv}$ and molecular docking for exploration of drugs J Mol Model. 2009; 15:453-460.

15. V Singh, P Somvanshi Toward the virtual screening of potential drugs in the homology modeled NAD+ dependent DNA ligase from Mycobacterium tuberculosis Protein and peptide letters 2010; 17(2):269-276.

16. Muralidharan, A. R., Selvaraj, C., Singh, S. K., Sheu, J. R., Thomas, P. A., \& Geraldine, P. Structure-based virtual screening and biological evaluation of a calpain inhibitor for prevention of selenite-induced cataractogenesis in an in vitro system. J ChemInf Model 2015; 55, 1686-97. doi: 10.1021/acs.jcim.5b00092.

17. Muralidharan, A. R., Selvaraj, C., Singh, S., Nelson, and Jesudasan, C. A., Geraldine, P., \& Thomas, P. Virtual screening based on pharmacophoric features of known calpain inhibitors to identify potent inhibitors of calpain. Med. Chem. Res. 2014, 23, 2445-2455.

18. Singh V and Somvanshi P Inhibition of Oligomerization of Aerolysin from Aeromonas Hydrophila: Homology Modeling and Docking Approach for Exploration of Hemorrhagic Septicemia. Letters in Drug Design \& Discovery. 2009; 6(3):215-223.

19. Lipinski, C. A., Lombardo, F., Dominy, B. W, \& Feeney, P. J. Experimental and computational approaches to estimate solubility and permeablility in drug discovery and development settings. Adv Drug Deliv Rev, 1997; 23, 3-25. doi:10.1016/S0169409X(96)00423-1.

20. Friesner, R. A., Murphy, R. B., Repasky, M. P., Frye, L. L., Greenwood, J. R., Halgren, T. A., Sanschagrin, P. C., \& Mainz, D. T. Extra precision glide: docking and scoring incorporating a model of hydrophobic enclosure for protein-ligand complexes. J. Med. Chem. 2006; 49, 6177-6196.

21. Fidele N. K. An in silico evaluation of the ADMET profile of the StreptomeDB database. SpringerPlus, 2013; 353, 1-11. doi: 10.1186/2193-1801-2-353

22. Wang, B., Zhao, J., \& Zhang, P. Gene signatures in osteoarthritic acetabular labrum using microarray analysis. International Journal of Rheumatic Diseases. 2016; 1-3.

23. Gortazar, A. R., Martin-Millan, M., Bravo, B., Plotkinm , L. I., \& Bellido, T. Crosstalk between caveolin-1/extracellular signal-regulated kinase (ERK) and catenin survival pathways in osteocyte mechanotransduction. JBC. 2013; 288, $8168-8175$ doi 10.1074/jbc.M112.437921

24. Berman, H.M., Battistuz, T., Bhat, T.N., Bluhm, W.F., Bourne, P.E., Burkhardt, K., Feng, Z., Gilliland, G.L., Iype, L., Jain, S., Fagan, P., Marvin, J., Padilla, D., Ravichandran, V., Schneider, B., Thanki, N., Weissig, H., \& Westbrook, J.D. The protein data bank. Acta Crystallogr D Biol Crystallogr 2002; 58, 899-907.

25. Zhang, Y. I-TASSER server for protein 3D structure prediction. BMC Bioinformatics, 2008; 9, 40.

26. Roy, A., Kucukural, A., \& Zhang, Y. I-TASSER: A unified platform for automated protein structure and function prediction. Nature Protocols, 2010; 5, 725-738.

27. Yang, J., \& Zhang, Y. I-TASSER server: New development for protein structure and function predictions. Nucleic Acids Research, 2015; 43, W174-W181.

28. Yang, J., Yan, R., Roy, A., Xu, D., Poisson, J., \& Zhang, Y. The I-TASSER Suite: Protein structure and function prediction. Nature Methods, 2015; 12, 7-8. 
29. Laskowski, R. A., MacArthur, M. W., Moss, D. S., \& Thornton, J. M. PROCHECK: A program to check the stereochemical quality of protein structures. J. App. Cryst., 1993; 26, 283-291.

30. Pontius, J., Richelle, J., \& Wodak, S. J. Deviations from standard atomic volumes as a quality measure for protein crystal structures. J Mol Biol., 1996; 264, 121-36.

31. Colovos, C., \& Yeates, T. O. Verification of protein structures: Patterns of nonbonded atomic interactions. Protein Sci.,1993; 2, 1511-1519.

32. Maestro, version 10.3, Schrodinger, LLC, New York, NY, 2015.

33. Irwin, J.J., Sterling, T., Mysinger, M.M., Bolstad, E.S., \& Coleman, R.G. ZINC: a free tool to discover chemistry for biology. J. Chem. Inf. Model. 2012; doi:10.1021/ci3001277.

34. Khanam U., Malik B. K., Mathur P, \& Rathi B. Identification of novel inhibitors for mitogen activated protein kinase kinase 4 by virtual screening and molecular dynamics simulation techniques. International Journal of Pharmaceutical Sciences. 2016; 8, 262268.

35. LigPrep, version 3.5, Schrodinger, LLC, New York, NY, 2015.

36. Epik version 3.3, Schrodinger, LLC, New York, NY, 2015

37. Glide, version 6.8, Schrodinger, LLC, New York, NY, 2015.

38. QikProp, version 4.5, Schrodinger, LLC, New York, NY, 2015.

39. Kroemer, R. T. Structure-Based Drug Design: Docking and Scoring: Current Protein and Peptide. Science, 2007; 8, 312-328.

40. Prime, version 4.1, Schrodinger, LLC, New York, NY, 2015.

41. Shivakumar, D., Williams, J., Wu, Y., Damm, W., Shelley, J., \& Sherman, W. Prediction of absolute solvation free energies using molecular dynamics free energy perturbation and the OPLS force field. J. Chem. Theory Comput. 2010; 6, 1509-1519. doi:10.1021/ct900587b

42. Branka, A. Nose-Hoover chain method for nonequilibrium molecular dynamics simulation. Phys. Rev. 2000; E 61, 4769-4773. doi:10.1103/PhysRevE.61.4769.

43. Martyna, G.J., Tobias, D.J., \& Klein, M.L. Constant pressure molecular dynamics algorithms. J. Chem. Phys. 1994; 101, 4177. doi:10.1063/1.467468.

44. Krautler, V. Van, G.W.F \& Philippe, H.H. A fast SHAKE algorithm to solve distance constraint equations for small molecules in molecular dynamics simulations. J. Comput. Chem. 2001; 22, 501-508.

\section{Figure Legends}

Figure 1. Protein 3D-structure model and validation results: (a) 3-D model rendered by ITasser server. (b) Ramachandran plot analysis. (c) Overall quality of the model evaluated by the ERRAT program. (d) Average Z-score is displayed in the plot.

Figure 2. Top-five hit compounds obtained from virtual screening, $2 \mathrm{D}$ structures and their corresponding ZINC IDs.

Figure 3. 2D-interaction map of top-five hit compounds obtained by induced fit docking (a) ZINC13410492, (b) ZINC08298670, (c) ZINC13550046, (d) ZINC01535903, and (e) ZINC13431926.

Figure 4. Illustrating RMSD plot obtained from 100 ns MD simulation run of ZINC13410492 system (magenta), and Cav-1 without ligand (blue); X and Y axes represent time (ns), and RMSD, respectively. 
Figure 5. Illustrating RMSF plot obtained from 100 ns MD simulation run of ZINC13410492 system RMSF plots for the MD simulations, showing the deviations by residue, of Cav-1. Blue line: atoms of the C-alpha; Green line: backbone; Standing line: ligand-residue contact, respectively.

Figure 6. Protein-ligand contact plot for 100 ns MD simulation of ZINC13410492 system shows binding interactions, hydrogen bond (green), ionic interaction (pink), water bridge (blue) and hydrophobic interaction (purple).

Figure 7. Protein-protein interaction network of Cav-1.

Table 1. Structure validation of the predicted model by PROCHECK, ERRAT and PROVE.

\begin{tabular}{|l|l|l|}
\hline \multicolumn{4}{|l|}{ Validation Index } & Cav-1 \\
\hline \multirow{4}{*}{ Ramachandran plot } & Residues in most favoured regions & $93.1 \%$ \\
\cline { 2 - 3 } & Residues in additional allowed regions & $3.8 \%$ \\
\cline { 2 - 3 } & Residues in generously allowed regions & $1.2 \%$ \\
\cline { 2 - 3 } & Residues in disallowed regions & $1.9 \%$ \\
\hline ERRAT & 72.941 \\
\hline Prove Z-Score RMS & 1.631 \\
\hline
\end{tabular}

Table 2. Top-five hit compounds obtained from high throughput virtual screening protocol and induced fit docking against Cav-1 along with Glide score, Glide energy, IFD score and MM/GBSA.

\begin{tabular}{|c|c|c|c|c|}
\hline Title & Glide energy & Glide score & $\begin{array}{c}\text { MM/GBSA Dg } \\
\text { binding }\end{array}$ & IFD score \\
\hline ZINC13410492 & -40.244 & -8.032 & -63.345 & -339.945 \\
\hline ZINC08298670 & -42.47 & -7.62 & -58.716 & -337.915 \\
\hline ZINC13550046 & -36.098 & -7.152 & -46.152 & -333.947 \\
\hline ZINC01535903 & -45.35 & -7.052 & -55.253 & -342.451 \\
\hline ZINC13431926 & -35.302 & -6.978 & -59.28 & -331.895 \\
\hline
\end{tabular}

Table 3. ADMET descriptors of IFD compounds

\begin{tabular}{|l|c|c|c|c|c|}
\hline Title & ZINC13410492 & ZINC08298670 & ZINC13550046 & ZINC01535903 & ZINC13431926 \\
\hline QPlogS & -3.589 & -1.74 & 2 & -0.562 & -1.482 \\
\hline QPlogPw & 9.698 & 15.444 & 11.068 & 15.599 & 9.102 \\
\hline QPlogPoct & 20.598 & 22.978 & 14.507 & 20.68 & 17.125 \\
\hline QPlogPo/w & 4.793 & 0.997 & -0.92 & -0.249 & 2.157 \\
\hline QPlogPC16 & 14.91 & 11.994 & 6.746 & 11.691 & 8.898 \\
\hline QPlogBB & -0.404 & -0.321 & -0.08 & -1.397 & 1.213 \\
\hline QPlogKp & -4.733 & -6.8 & -7.351 & -8.624 & -5.951 \\
\hline QPlogHERG & -8.23 & -5.589 & -5.735 & -6.508 & -5.864 \\
\hline QPpolrz & 42.35 & 38.996 & 18.448 & 31.455 & 37.149 \\
\hline
\end{tabular}




\begin{tabular}{|c|c|c|c|c|c|}
\hline QPPMDCK & 41.275 & 26.677 & 4.756 & 1.204 & 59.767 \\
\hline QPPCaco & 83.341 & 24.15 & 10.281 & 3.168 & 106.907 \\
\hline QPlogKhsa & 0.884 & -0.332 & -0.662 & -0.388 & 0.574 \\
\hline $\begin{array}{l}\text { Percent Human } \\
\text { Oral } \\
\text { Absorption }\end{array}$ & 89.386 & 57.534 & 39.671 & 21.494 & 75.89 \\
\hline SASA & 740.934 & 672.718 & 440.355 & 582.99 & 587.978 \\
\hline FOSA & 265.338 & 301.617 & 316.495 & 142.623 & 571.359 \\
\hline FISA & 91.621 & 135.243 & 123.86 & 241.372 & 16.619 \\
\hline $\mathrm{EA}(\mathrm{eV})$ & -0.217 & 0.227 & -2.52 & 1.643 & -2.421 \\
\hline $\mathrm{IP}(\mathrm{eV})$ & 9.283 & 9.316 & 9.189 & 8.728 & 8.748 \\
\hline CNS & 0 & 0 & 0 & -2 & 2 \\
\hline SAfluorine & 0 & 47.02 & 0 & 0 & 0 \\
\hline SAamideO & 0 & 15.612 & 0 & 0 & 0 \\
\hline Rule of Five & 0 & 0 & 0 & 1 & 0 \\
\hline PSA & 49.272 & 97.487 & 66.017 & 138.512 & 25.499 \\
\hline PISA & 383.976 & 188.837 & 0 & 198.995 & 0 \\
\hline $\begin{array}{l}\text { Human Oral } \\
\text { Absorption }\end{array}$ & 1 & 2 & 2 & 1 & 3 \\
\hline glob & 0.7889629 & 0.8097446 & 0.8748836 & 0.8409039 & 0.8668947 \\
\hline CIQPlogS & -3.627 & -1.515 & 1.996 & -1.554 & -0.57 \\
\hline \#stars & 1 & 0 & 2 & 1 & 3 \\
\hline
\end{tabular}

(C) 2016 by the authors; licensee Preprints, Basel, Switzerland. This article is an open access article distributed under the terms and conditions of the Creative Commons by Attribution (CC-BY) license (http://creativecommons.org/licenses/by/4.0/). 